\title{
Path Analysis of Characteristics of Panchayat Leaders and its Impact on their Role Performance
}

\author{
P. Shrivastava ${ }^{1^{*}}$ and K.K. Shrivastava ${ }^{2}$ \\ ${ }^{1}$ (Ag. Extn.), College of Agriculture, Balaghat (JNKVV, Jabalpur), India \\ ${ }^{2}$ (Ag. Extn.), Directorate of Extension Services, IGKV, Raipur, India \\ *Corresponding author
}

\section{Keywords \\ Panchayati Raj \\ leaders, \\ Characteristics, \\ Impact and path \\ analysis}

Article Info

Accepted:

15 January 2018

Available Online:

10 February 2018

\section{A B S T R A C T}

A good deal of work in establishing closer linkage between research and extension has been done in the last few years. The result of painstaking efforts of research scientists have been carried to the farmers by an army of more than 70,000 village extension workers in the major states where professional agricultural extension system is functioning. As defined by Roling (1988) extension is purposeful communication intervention deployed by an institution to induce change in voluntary behaviours with a presumed public or collective utility. The $73^{\text {rd }}$ Amendment provides for an elaborate system of establishing panchayats as units of self-government. There are more than two lakh panchayats across the country represented by more than 30 lakh of elected leaders at all three levels. Out of them, more than six lakh SC/ST's and more than ten lakh women have been holding the office of the members and chairpersons at different tiers of the panchayats. A study was conducted in the purposively selected Rajnandgaon district of Chhattisgarh to ascertain the attitude of panchayat leaders towards the Panchayati Raj Institutions. In all $(9+54+200)$ 263 panchayat leaders were considered as respondents for the study. An interview schedule was used as a tool for collecting primary data from the respondent panchayat leaders and all the 263 respondents were personally interviewed for collecting the raw information. The most important variable which had negative substantial indirect effect on role performance of gram panchayat leaders was age (-0.1062) through attitude towards panchayati raj institutions. The variable achievement motivation had highest negative substantial indirect effect (-0.6237) through socio-economic status on role performance of janpad and jila panchayat leaders followed by social participation $(-0.2140)$ through social participation.

\section{Introduction}

India's past success under the Green Revolution now requires a further impetus through a two component strategy: to further improve on performance of agricultural systems already influenced by the Green Revolution, and expansion of productivity growth in the much larger segments which did not witness Green Revolution. A good deal of work in establishing closer linkage between research and extension has been done in the last few years. The result of painstaking efforts of research scientists have been carried to the farmers by an army of more than 70,000 village extension workers in the major states 
where professional agricultural extension system is functioning. A basic function of extension is to assist the transfer of agricultural technology by ensuring that an adequate amount of high quality knowledge about it is present in the farming community for sustained agricultural development. As defined by Roling (1988) extension is purposeful communication intervention deployed by an institution to induce change in voluntary behaviours with a presumed public or collective utility. It is difficult for any country to provide enough number of extension workers to reach each and every family for its social welfare programme. It is rather more difficult for the developing countries where the resources are scarce. It is, therefore, not possible to have face to face contact with each and every individual living in the villages. This problem can be solved to some extent through the use of local leaders. A local leader who has adopted improved practices extends the same to others. The common man has much faith in the local leaders. The concept of leadership has undergone considerable change as research in this area of study progressed. Leadership was considered at one time to be a set of traits handed down chiefly through heredity from one person to another. Thus, positions of leadership were restricted to individuals belonging to certain strata of society commonly deemed worthy and suitable as leaders - leadership usually being determined by birth in the right family. It was discovered, however, as social and economic barriers disappeared, that leaders began to emerge from hitherto "restricted" strata of society and that leadership was not the monopoly of a few favoured familial groups or social strata. The $73^{\text {rd }}$ Amendment provides for an elaborate system of establishing panchayats as units of self-government. For the first time in the constitutional history of India, the constitution of panchayat, the duration of their term, their membership, the constitution of Finance
Commission to review their financial position is detailed. It also adds a new schedule to the Constitution the Eleventh Schedule, which lists 29 subjects, which are to be handled by the panchayats. With this Amendment, Panchayati Raj institutions are as much a part of the structure of constitutional government in India as the Lok Sabha. There are more than two lakh panchayats across the country represented by more than 30 lakh of elected leaders at all three levels. Out of them more than six lakh SC/ST's and more than ten lakh women have been holding the office of the members and chairpersons at different tiers of the panchayats. This is unparallel across the world. Given this, let us discuss the role of the panchayati raj institutions. In general panchayats should be able to facilitate member work effectiveness by influencing strategy choices more for complex jobs than for simple, straight forward or routine ones. The reason is that on simple jobs, strategy choices usually cannot make much of a difference in effectiveness; instead, how well one does is determined almost entirely by how hard one works. On jobs characterised by high variety and autonomy, on the other hand, the work strategy used by the individual usually is of considerable importance in determining work effectiveness. With the above points of reference in view a research was planned to explore the following objective:

To predict the variation in performance (path analysis) of panchayat leaders caused by independent variables (characteristics of panchayat leaders).

\section{Materials and Methods}

Research methodology is a detailed action plan of investigation. It describes and clarifies methods used for measuring dependent and independent variables as well as techniques followed for the collection and analysis of data. A study was conducted in the 
purposively selected Rajnandgaon district of Chhattisgarh to ascertain the attitude of panchayat leaders towards the Panchayati Raj Institutions. Out of total 21 members of the Jila Panchayat including the president, 9 members who presided over the different standing committees were purposively selected as respondents from the first tier panchayat. Similarly out of the total 182 members of the 9 janpad panchayats, 6 members from each janpad $(9 \times 6=54)$ presiding over the standing committees were purposively selected as respondents from second tier panchayats.

In all there are 696 gram panchayats in 9 blocks/janpads of Rajnandgaon district. Approximately seven per cent gram panchayats were randomly selected from each janpad and four members presiding over the standing committees from each selected gram panchayat $(50 \times 4=200)$ were purposively chosen as respondents from the third tier panchayats.

Thus in all $(9+54+200) 263$ panchayat leaders were considered as respondents for the study. An interview schedule was used as a tool for collecting primary data from the respondent panchayat leaders and all the 263 respondents were personally interviewed for collecting the raw information.

To know the direct and indirect effects of various independent variables on the dependent variables (Attitude and Role performance), the method of path coefficient analysis (Wrights, 1921) was employed.

Path coefficient technique is an extension of the technique of standard and partial regression coefficient. Path effects were obtained by solving the simultaneous equations set up for the purpose using the correlation matrix. Considering

$x_{1}$ independent variable to be influencing the dependent variable $y_{1}$ the simultaneous equation would be:

$$
r y x_{i}=P_{y x_{i}} \times \sum_{i, j=1}^{n} r x_{i} x_{j} \times P_{y x_{i}}
$$

Where,

$r y x_{i}$ is the correlation coefficient of ${ }^{x_{i}}$ with $y$

$P_{y x_{i}}$ is the direct effect and each of the other term in the equation is an indirect effect.

$\sum_{i, j=1}^{n} r x_{i} x_{j} \times P_{y x_{i}}$ is the indirect effect of independent variable to $i, j=1$ dependent variable via another independent variable.

\section{Results and Discussion}

Direct, total indirect and substantial indirect effects of independent variables on role performance of gram panchayat leaders

\section{Direct effect}

The data in table 1 reveals that the variable material possession exerted the highest positive direct effect on role performance of gram panchayat leaders as the path coefficient value was 0.3742 , followed by attitude towards panchayati raj institutions (0.3222), education (0.2804), extension participation (0.2750), cosmopoliteness (0.0773), size of land holding (0.0627), information sources (0.0498), achievement motivation (0.0380), occupation (0.0354), political ideology (0.0132), political affiliation (0.0054) and family size (0.0009) whereas the variable socio economic status had maximum negative direct effect on role performance of gram panchayat leaders with path coefficient - 
0.2863 , followed by political efficacy ($0.0650)$, social participation (- 0.0468), age ($0.0467)$, caste $(-0.0342)$, time allocation for panchayat activities (-0.0329), annual income $(-0.0297)$, training need $(-0.0202)$, gender $(-$ 0.0195), experience (- 0.0091) and job satisfaction (- 0.0011).

The discussion leads to the conclusion that the variable material possession exerted the highest positive direct effect on role performance of gram panchayat leaders.

The other variables with positive direct effect on role performance in sequential order are: attitude towards panchayati raj institutions, education, extension participation, cosmopoliteness, size of land holding, information sources, achievement motivation, occupation, political ideology, political affiliation and family size.

Whereas the variables with negative direct effect in sequential order are socio economic status, political efficacy, social participation, age, caste, time allocation for panchayat activities, annual income, training need, gender, experience and job satisfaction.

\section{Total indirect effect}

It is obvious from the table 1 that variable socio economic status had highest positive total indirect effect with path coefficient 0.7400 followed by cosmopoliteness (0.6227), extension participation (0.5747), attitude towards panchayati raj institutions (0.5495), education (0.5037), time allocation for panchayat activities (0.3239), annual income (0.3082), size of land holding (0.3043), information sources (0.2701), occupation (0.3170), job satisfaction (0.1965), caste (0.1676), political efficacy (0.1662), achievement motivation (0.1643), family size (0.1437), political ideology (0.1266), political affiliation (0.1258), training need (0.1217), experience (0.1192), material possession (0.0541) and social participation (0.0535). While the variable age had highest negative total indirect effect $(-0.2887)$ followed by gender (- 0.0592).

From the above discussion it can be concluded that the variable socio economic status showed highest positive total indirect effect followed by cosmopoliteness, extension participation attitude towards panchayati raj institutions, education, time allocation for panchayati raj activities, annual income, size of land holding, information sources, occupation, job satisfaction, caste, political efficacy, achievement motivation, family size, political ideology, political affiliation, training need, experience, material possession and social participation. Only two variables age and gender had negative total indirect effect on role performance of gram panchayat leaders.

\section{Substantial indirect effect}

It is clear from the table 1 that the variable material possession had the highest positive substantial indirect effect $(0.3742)$ on role performance through itself followed by socio economic status (0.3682) through material possession, attitude towards panchayati raj institutions (0.3222) through itself, education (0.2804) through itself, extension participation (0.2750) through itself, size of land holding (0.2704) through material possession, annual income (0.2338) through material possession, cosmopoliteness (0.2104) through attitude, time allocation for panchayat activities (0.1163) through material possession, political efficacy (0.1128) through education, occupation (0.0991) through material possession, information sources (0.0944) through attitude, caste (0.0705) through material possession, achievement motivation (0.0559) through attitude, job satisfaction (0.0536) through attitude, political ideology (0.0494) through education, political 
affiliation (0.0460) through attitude, of gram panchayat leaders were age (- 0.1062) experience (0.0435) through extension through attitude towards panchayati raj participation and training need (0.0320) institutions, family size (- 0.0899) through through attitude towards panchayati raj socio economic status, gender (- 0.0513) institutions. The variables which had negative through education and social participation (substantial indirect effect on role performance 0.0468) through social participation itself.

Table.1 Path coefficient showing direct, indirect and substantial indirect effect of independent variables on role performance of gram panchayat leaders

\begin{tabular}{|c|c|c|c|c|c|}
\hline $\begin{array}{l}\text { S. } \\
\text { N } \\
\text { o. }\end{array}$ & $\begin{array}{l}\text { Va } \\
\text { ria } \\
\text { ble }\end{array}$ & Independent Variables & $\begin{array}{l}\text { Direct } \\
\text { Effect }\end{array}$ & $\begin{array}{c}\text { Total } \\
\text { Indirect } \\
\text { Effect }\end{array}$ & $\begin{array}{c}\text { Substantial } \\
\text { indirect } \\
\text { effect } \\
\text { through first }\end{array}$ \\
\hline 1 & $\mathrm{X}_{1}$ & Age & -0.0467 & -0.2887 & $-0.1062\left(X_{23}\right)$ \\
\hline 2 & $\mathrm{X}_{2}$ & Gender & -0.0195 & -0.0592 & $-0.0513\left(\mathrm{X}_{4}\right)$ \\
\hline 3 & $\mathrm{X}_{3}$ & Caste & 0.0342 & 0.1876 & $0.0705\left(\mathrm{X}_{10}\right)$ \\
\hline 4 & $\mathrm{X}_{4}$ & Education & 0.2804 & 0.5037 & $0.2804\left(\mathrm{X}_{4}\right)$ \\
\hline 5 & $\mathrm{X}_{5}$ & Family size & 0.0009 & 0.1437 & $-0.0899\left(X_{11}\right)$ \\
\hline 6 & $\mathrm{X}_{6}$ & Social participation & -0.0468 & 0.0535 & $-0.0468\left(X_{6}\right)$ \\
\hline 7 & $\mathrm{X}_{7}$ & Occupation & 0.0354 & 0.2170 & $0.0991\left(\mathrm{X}_{10}\right)$ \\
\hline 8 & $\mathrm{X}_{8}$ & Annual Income & -0.0297 & 0.3082 & $0.2338\left(\mathrm{X}_{10}\right)$ \\
\hline 9 & $\mathrm{X}_{9}$ & Size of land holding & 0.0627 & 0.3043 & $0.2704\left(\mathrm{X}_{10}\right)$ \\
\hline 10 & $\mathrm{X}_{10}$ & Material possession & 0.3742 & 0.0541 & $0.3742\left(\mathrm{X}_{10}\right)$ \\
\hline 11 & $\mathrm{X}_{11}$ & Socio economic status & -0.2863 & 0.7400 & $0.3682\left(\mathrm{X}_{10}\right)$ \\
\hline 12 & $\mathrm{X}_{12}$ & Achievement motivation & 0.0380 & 0.1643 & $0.0559\left(\mathrm{X}_{23}\right)$ \\
\hline 13 & $\mathrm{X}_{13}$ & Cosmopoliteness & 0.0773 & 0.6227 & $0.2104\left(X_{23}\right)$ \\
\hline 14 & $\mathrm{X}_{14}$ & Job satisfaction & -0.0011 & 0.1965 & $0.0536\left(\mathrm{X}_{23}\right)$ \\
\hline 15 & $\mathrm{X}_{15}$ & Information sources & 0.0498 & 0.2701 & $0.0944\left(X_{23}\right)$ \\
\hline 16 & $\mathrm{X}_{16}$ & Extension participation & 0.2570 & 0.5747 & $0.2750\left(\mathrm{X}_{16}\right)$ \\
\hline 17 & $\mathrm{X}_{17}$ & $\begin{array}{l}\text { Time allocation to panchayat } \\
\text { activities }\end{array}$ & -0.0329 & 0.3239 & $0.1163\left(X_{10}\right)$ \\
\hline 18 & $\mathrm{X}_{18}$ & Experience & -0.0091 & 0.1192 & $0.0435\left(\mathrm{X}_{16}\right)$ \\
\hline 19 & $\mathrm{X}_{19}$ & Training need & -0.0202 & 0.1217 & $0.0320\left(X_{23}\right)$ \\
\hline 20 & $\mathrm{X}_{20}$ & Political affiliation & 0.0054 & 0.1258 & $0.0460\left(\mathrm{X}_{23}\right)$ \\
\hline 21 & $\mathrm{X}_{21}$ & Political ideology & 0.0132 & 0.1266 & $0.0494\left(X_{4}\right)$ \\
\hline 22 & $\mathrm{X}_{22}$ & Political efficacy & -0.0650 & 0.1662 & $0.1128\left(\mathrm{X}_{4}\right)$ \\
\hline 23 & $\mathrm{X}_{23}$ & $\begin{array}{l}\text { Attitude towards panchayati } \\
\text { raj institutions }\end{array}$ & 0.3222 & 0.5495 & $0.3222\left(\mathrm{X}_{23}\right)$ \\
\hline
\end{tabular}


Table.2 Path coefficient showing direct, indirect and substantial indirect effect of independent variables on role performance of janpad and jila panchayat leaders

\begin{tabular}{|r|r|l|r|r|r|}
\hline $\begin{array}{c}\text { S. } \\
\text { No. }\end{array}$ & Variable & Independent Variables & Direct Effect & $\begin{array}{c}\text { Total } \\
\text { Indirect } \\
\text { Effect }\end{array}$ & $\begin{array}{c}\text { Substantial } \\
\text { indirect effect } \\
\text { through first }\end{array}$ \\
\hline 1 & $\mathrm{X}_{1}$ & Age & -0.0678 & -0.1834 & $0.2069\left(\mathrm{X}_{11}\right)$ \\
\hline 2 & $\mathrm{X}_{2}$ & Gender & 0.0562 & -0.0279 & $-0.1978\left(\mathrm{X}_{11}\right)$ \\
\hline 3 & $\mathrm{X}_{3}$ & Caste & -0.0216 & 0.2782 & $0.6513\left(\mathrm{X}_{11}\right)$ \\
\hline 4 & $\mathrm{X}_{4}$ & Education & -0.2443 & 0.5007 & $0.8593\left(\mathrm{X}_{11}\right)$ \\
\hline 5 & $\mathrm{X}_{5}$ & Family size & -0.2140 & 0.3299 & $1.0879\left(\mathrm{X}_{11}\right)$ \\
\hline 6 & $\mathrm{X}_{6}$ & Social participation & -0.1642 & 0.3453 & $-0.2140\left(\mathrm{X}_{6}\right)$ \\
\hline 7 & $\mathrm{X}_{7}$ & Occupation & -.1841 & 0.1660 & $1.4031\left(\mathrm{X}_{11}\right)$ \\
\hline 8 & $\mathrm{X}_{8}$ & Annual Income & -1.6709 & 0.9171 & $2.0226\left(\mathrm{X}_{11}\right)$ \\
\hline 9 & $\mathrm{X}_{9}$ & Size of land holding & 2.6931 & -2.2702 & $2.6490\left(\mathrm{X}_{11}\right)$ \\
\hline 10 & $\mathrm{X}_{10}$ & Material possession & 0.1937 & -0.0869 & $-0.6931\left(\mathrm{X}_{11}\right)$ \\
\hline 11 & $\mathrm{X}_{11}$ & Socio economic status & 0.2312 & 0.5365 & $0.7336\left(\mathrm{X}_{11}\right)$ \\
\hline 12 & $\mathrm{X}_{12}$ & Achievement motivation & -0.0172 & 0.0821 & $0.2441\left(\mathrm{X}_{11}\right)$ \\
\hline 13 & $\mathrm{X}_{13}$ & Cosmopoliteness & 0.0572 & 0.2169 & $1.2740\left(\mathrm{X}_{11}\right)$ \\
\hline 14 & $\mathrm{X}_{14}$ & Job satisfaction & 0.2678 & 0.4665 & $1.2550\left(\mathrm{X}_{11}\right)$ \\
\hline 15 & $\mathrm{X}_{15}$ & Information sources & -0.1062 & 0.3402 & $1.3920\left(\mathrm{X}_{11}\right)$ \\
\hline 16 & $\mathrm{X}_{16}$ & Extension participation & & & \\
\hline 17 & $\mathrm{X}_{17}$ & Time allocation to panchayat & -0.1125 & 0.1043 & $-0.1125\left(\mathrm{X}_{18}\right)$ \\
\hline & & activities & -0.0687 & -0.1148 & $0.3466\left(\mathrm{X}_{11}\right)$ \\
\hline 18 & $\mathrm{X}_{18}$ & Experience & -0.0440 & 0.0042 & $0.4237\left(\mathrm{X}_{11}\right)$ \\
\hline 19 & $\mathrm{X}_{19}$ & Training need & -0.0898 & 0.1463 & $-0.1325\left(\mathrm{X}_{11}\right)$ \\
\hline 20 & $\mathrm{X}_{20}$ & Political affiliation & -0.0742 & 0.0481 & $1.1339\left(\mathrm{X}_{11}\right)$ \\
\hline 21 & $\mathrm{X}_{21}$ & Political ideology & 0.1259 & 0.5368 & $0.9149\left(\mathrm{X}_{11}\right)$ \\
\hline 22 & $\mathrm{X}_{22}$ & Political efficacy & & \\
\hline 23 & $\mathrm{X}_{23}$ & Attitude towards panchayati & & \\
\hline & & raj institutions & & \\
\hline & & & & \\
\hline
\end{tabular}

Direct, total indirect and substantial indirect effect of independent variables on role performance of janpad and jila panchayat leaders

\section{Direct effect}

The path coefficient shown in table 2 reveal that the variable socio economic status has maximum positive direct effect (2.6931) on the role performance of janpad and jila panchayat leaders, followed by extension participation (0.2678), education (0.2443), cosmopoliteness (0.2318), achievement motivation (0.1937), annual income (0.1841), attitude towards panchayati raj institutions (0.1259), information sources (0.0572) and gender (0.0562).

The independent variables which had negative direct effect on the role performance of janpad and jila panchayat leaders, in the sequential order are material possession (1.9173), size of land holding (-0.6709), family size (-0.2927), social participation ($0.2140)$, occupation (-0.1642), experience (- 
0.1125), time allocation for panchayat activities (-0.1062), political ideology (0.0898), political efficacy (-0.0742), training need $(-0.0687)$, age $(-0.0678)$, political affiliation $(-0.0440)$, caste $(-0.0216)$ and job satisfaction (0.0172).

\section{Total indirect effect}

It is observed from the data in table 2 that the variable material possession had maximum total indirect effect (2.2702) on the role performance of janpad and jila panchayat leaders followed by size of land holding (0.9171), attitude towards panchayati raj institutions (0.5368), cosmopoliteness (0.5365), education (0.5007), extension participation (0.4665), social participation (0.4453), occupation (0.3615), time allocation for panchayat activities (0.3402), family size (0.3299), caste $(0.2782)$, information sources (0.2169), annual income (0.1660), political ideology (0.1463), experience $(0.1043)$, job satisfaction (0.0821), political efficacy (0.0481) and political affiliation (0.0042).

Only five variables viz. socio economic status $(-2.3342)$, age $(-0.1834)$, training need ($0.1148)$, achievement motivation (-0.0869) and gender (-0.0279) had negative total indirect effect on the role performance of janpad and jila panchayat leaders.

\section{Substantial indirect effect}

The data given in table 2 also indicate that the independent variable socio economic status had maximum positive substantial indirect effect (2.6931) through socio economic status itself.

This was followed by material possession (2.6490) through socio economic status, size of land holding (2.0226) through socio economic status, occupation (1.4031) through socio economic status, time allocation for panchayat activities (1.3920) through socio economic status, annual income (1.2813) through socio economic status, information sources (1.2740) through socio economic status, political efficacy (1.1339) through socio economic status, extension participation (1.1250) through socio economic status, family size (1.0879) through socio economic status, attitude towards panchayati raj institutions (0.9149) through socio economic status, education (0.8593) through socio economic status, cosmopoliteness (0.7336) through socio economic status, caste (0.6513) through socio economic status, political affiliation (0.4237) through socio economic status, training need (0.3466) through socio economic status, job satisfaction (0.2441) through socio economic status and age (0.2069) through socio economic status.

However, the variable achievement motivation had highest negative substantial indirect effect (-0.6237) through socioeconomic status on role performance of janpad and jila panchayat leaders followed by social participation $(-0.2140)$ through social participation, gender $(-0.1978)$ through socio economic status, political ideology $(-0.1325)$ through socio economic status and experience $(-0.1125)$ through experience itself.

The variable material possession exerted the highest positive direct effect on role performance of gram panchayat leaders as the path coefficient value was 0.3742 , followed by attitude towards panchayati raj institutions (0.3222), education (0.2804) and extension participation (0.2750) whereas the variable socio economic status had maximum negative direct effect on role performance of gram panchayat leaders with path coefficient 0.2863. The variable socio economic status had highest positive total indirect effect with path coefficient 0.7400 followed by cosmopoliteness (0.6227), extension participation (0.5747), attitude towards 
panchayati raj institutions (0.5495) and education (0.5037) while the variable age had highest negative total indirect effect ($0.2887)$ followed by gender (- 0.0592). The variable material possession had the highest positive substantial indirect effect (0.3742) on role performance through itself followed by socio economic status (0.3682) through material possession and attitude towards panchayati raj institutions (0.3222) through itself.

The most important variable which had negative substantial indirect effect on role performance of gram panchayat leaders was age (- 0.1062) through attitude towards panchayati raj institutions

The variable socio economic status has maximum positive direct effect (2.6931) on the role performance of janpad and jila panchayat leaders, followed by extension participation (0.2678), education (0.2443) and cosmopoliteness (0.2318). The independent variable which had negative direct effect on the role performance of janpad and jila panchayat leaders, in the sequential order are material possession(-1.9173), size of land holding (-0.6709), family size (- 0.2927) and social participation(- 0.2140). The variable material possession had maximum total indirect effect (2.2702) on the role performance of janpad and jila panchayat leaders followed by size of land holding (0.9171) and attitude towards panchayati raj institutions (0.5368). The variable socio economic status $(-2.3342)$ had negative total indirect effect on the role performance of janpad and jila panchayat leaders. The independent variable socio economic status had maximum positive substantial indirect effect (2.6931) through socio economic status itself.

The variable achievement motivation had highest negative substantial indirect effect (-
0.6237) through socio-economic status on role performance of janpad and jila panchayat leaders followed by social participation (0.2140 ) through social participation.

\section{References}

Babu, G.R. (2008) Research methodology in social sicences, Concept Publishing Company, New Delhi.

Baldoni, J. (2006) How great leaders get results, Tata McGraw Hill Publishing Company Ltd., New Delhi.

Bareth, L.S. and Sarkar, J.D. (2003) Role of opinion leaders in rural development and community advancement, Manage Extension Research Review, 67-73.

Chalapathi, B.V. and Raghavulu, B.V. (2005) Decline of good governance Decentralisation, Third Concept, August, 2005: 39-41.

Dashora, K.B. (2008) Women empowerment and panchayati raj, Third Concept, December 2008: 54-58.

Hooja, R. (2008) Capacity building for Rajasthan's panchayat representatives and functionaries; What the training efforts should cover, Indian J. Public Adminstration, 54(1): 1-20.

Kaur, G. (2003) Political dynamics of panchayati raj in India, Third Concept, September 2003: 17-25.

Khalge, M.I., Chole, R.R. and Bhosale, R.R. (2010) Role performance of gram panchayat members, Agriculture Update, 5 (1\&2): 47-51.

Lalneihzovi (2009) Decentralised governance for rural development in Mizoram: Problems and prospects, Indian J. Soc. Dev., 9(1):59-66.

Limbadri, R. (2007) Grass root democracy: The experience of Dalits in P.R.I.'s, Indian Journal of Public Administration, 53(4): 788-796.

Pachouri, P. (2007) A study of role performance of women panchayat 
leaders in village development of Narsinghpur district of M.P., Unpublished M.Sc. (Ag) thesis, JNKVV, Jabalpur.

Radhakrishnan, M.G. (2010) Action heroes The citizens who can and do, India Today - special issue, July 26:94.

Samad, M.A. (2007) Women empowerment and panchayati raj institutions in Kerala, Kurukshetra, March 2007:37-41.

Shrivastava M. (2010) Action heroes - The citizens who can and do, India Today special issue, July 26:96-97.

Shrivastava, K.K. (1999) Role perception and role performance of formal leaders working under panchayati raj system in Kheda district of Gujarat state, Unpublished Ph.D. Thesis, Gujarat
Agricultural University, Anand Campus, Anand.

Shrivastava, P. (2003) Job performance of village panchayat leaders of Jabalpur block, district Jabalpur, M.P., Unpublished M.Sc. (Ag) thesis, JNKVV, Jabalpur.

Shrivastava, P. (2011) An analytical study on role performance of leaders in the three tier Panchayati Raj Institutions of Rajnandgaon district of Chhattisgarh., Unpublished Ph.D.(Ag) thesis, IGKV, Raipur.

Shukla, S. (2009) A study on performance of entrepreneurial activities among the members of women self-help groups (SHGs) in Dharamjaigarh block of Raigarh district (C.G.), Unpublished M.Sc. (Ag) thesis, IGKV, Raipur.

\section{How to cite this article:}

Shrivastava, P. and Shrivastava, K.K. 2018. Path Analysis of Characteristics of Panchayat Leaders and its Impact on their Role Performance. Int.J.Curr.Microbiol.App.Sci. 7(02): 15871595. doi: https://doi.org/10.20546/ijcmas.2018.702.191 